\title{
BMJ Open Associations between urinary cysteine- rich protein 61 excretion and kidney function decline in outpatients with chronic kidney disease: a prospective cohort study in Taiwan
}

\author{
Chun-Fu Lai (D) , Jian-Jhong Wang, ${ }^{2}$ Ya-Chun Tu, ${ }^{1}$ Chia-Yu Hsu, ${ }^{3}$ \\ Hon-Yen Wu, ${ }^{1,4,5,6}$ Cheng-Chung Fang (D) , ${ }^{1,7}$ Yung-Ming Chen, ${ }^{1,8}$ Ming-Shiou Wu, ${ }^{1}$ \\ Tun-Jun Tsai ${ }^{1}$
}

To cite: Lai C-F, Wang J-J, Tu Y-C, et al. Associations between urinary cysteinerich protein 61 excretion and kidney function decline in outpatients with chronic kidney disease: a prospective cohort study in Taiwan. BMJ Open 2021;11:e051165. doi:10.1136/ bmjopen-2021-051165

- Prepublication history and additional supplemental material for this paper are available online. To view these files, please visit the journal online (http://dx.doi.org/10.1136/ bmjopen-2021-051165).

Received 14 March 2021 Accepted 20 September 2021

Check for updates

(C) Author(s) (or their employer(s)) 2021. Re-use permitted under CC BY-NC. No commercial re-use. See rights and permissions. Published by BMJ.

For numbered affiliations see end of article.

\section{Correspondence to} Dr Hon-Yen Wu; honyenwu@ntu.edu.tw and Dr Cheng-Chung Fang; conrad@ntu.edu.tw

\section{ABSTRACT}

Objectives To examine whether urinary excretion of cysteine-rich protein 61 (Cyr61), an acknowledged proinflammatory factor in kidney pathologies, increases in chronic kidney disease (CKD) and is associated with subsequent rapid kidney function decline.

Design An observational cohort study.

Setting In the nephrology outpatient clinics of a tertiary hospital in Taiwan.

Participants We enrolled 138 adult CKD outpatients $(n=12,32,18,18,29$ and 29 in stages 1, 2, 3a, 3b, 4 and 5 CKD, respectively) between February and October 2014 and followed them for 1 year. Their mean age was $60.46 \pm 13.16$ years, and 51 (37\%) of them were women. Primary outcome measures Urinary Cyr61 levels were measured by ELISA. Rapid kidney function decline was defined as an estimated glomerular filtration rate (eGFR) decline rate $\geq 4 \mathrm{~mL} / \mathrm{min} / 1.73 \mathrm{~m}^{2} /$ year or developing endstage renal disease during subsequent 3-month or 1-year follow-up period. Models were adjusted for demographic and clinical variables.

Results The urine Cyr61-to-creatinine ratio (UCyr61CR) increased significantly in patients with stage 4 or 5 CKD. Multivariable linear regression analysis showed that $\log (\mathrm{UCy}$ r61CR) was positively correlated with log(urine protein-to-creatinine ratio) $(p<0.001)$ but negatively correlated with baseline eGFR $(p<0.001)$ and hypertension $(p=0.007)$. Complete serum creatinine data during the follow-up were available for 112 patients (81.2\%). Among them, multivariable logistic regression identified log(UCyr61CR) was independently associated with rapid kidney function decline (adjusted OR 2.29, 95\% Cl 1.27 to 4.15$)$ during the subsequent 3 months. UCyr61CR improved the discriminative performance of clinical models to predict 3-month rapid kidney function decline. In contrast, log(UCyr61CR) was not associated with rapid eGFR decline during the entire 1-year follow-up. Conclusions Elevated urinary Cyr61 excretion is associated with rapid short-term kidney function deterioration in patients with CKD. Measuring urinary Cyr61 excretion is clinically valuable for monitoring disease trajectory and may guide treatment planning.
Strengths and limitations of this study

- This urinary biomarker-based research enrolled well-documented chronic kidney disease (CKD) outpatients across a wide range of glomerular filtration rate (GFR) and was representative for clinical practice.

- Substituting non-detectable urinary cysteine-rich protein 61 values to one-half the limit-of-detection of the assay leads to a negative bias for variability and does not estimate the mean well.

- Rapid GFR decline might also result from acute conditions affecting kidney function before or after enrolment, rather than CKD progression itself.

- A patient with CKD may have non-linear disease trajectories so that using two serum creatinine measurements to estimate disease progression may not be accurate.

- This moderately sized observational cohort study was unable to fully adjust for all potential confounders and does not imply causality.

\section{INTRODUCTION}

Chronic kidney disease (CKD) is threatening human health and economic burden worldwide. ${ }^{1}$ Treatment with renin-angiotensin-aldosterone system inhibitors in a multidisciplinary care setting is currently the mainstay therapy. ${ }^{2-4}$ Previous cohort studies showed the estimated glomerular filtration rate (eGFR) decline rate ranged from 1.02 to $3.76 \mathrm{~mL} / \mathrm{min} / 1.73 \mathrm{~m}^{2} /$ year in patients under the CKD care programme in Taiwan. ${ }^{35}$ This indicated that the ultimate goal of completely halting kidney disease pathogenesis is not achieved. The resultant advanced CKD and end-stage renal disease (ESRD) are associated with substantially increased morbidity and excess mortality. ${ }^{16}$ Identifying prognostic 
markers may help to identify individuals at risk of progression as early as possible. Such clinical markers may also help us to understand the pathological mechanisms and to develop novel disease-modifying therapies.

Sustained inflammation, hypoxia and fibrosis compose the common pathogenic pathway for most aetiologies of CKD. ${ }^{7}$ Several studies have shown that many inflammatory molecules display enhanced expression in CKD and that anti-inflammatory treatments have renoprotective effects. ${ }^{8-10}$ Unbiased transcriptional analyses also identified a variety of proinflammatory immune responses that were upregulated during progressive kidney disease. ${ }^{11-14}$ Furthermore, recent clinical investigations revealed that circulating inflammatory proteins are strongly associated with CKD progression. ${ }^{15} 16$ These data suggest that persistent activated inflammatory cascades participate in the pathogenesis of progressive kidney failure.

Cysteine-rich protein 61 (Cyr61), also known as CCN1, is a secreted matricellular protein belonging to the CCN protein family. ${ }^{17}$ Cyr61 has been reported to mediate various biological functions, including angiogenesis, apoptosis, tumourigenesis, matrix remodelling and fibrosis. ${ }^{18}$ Several lines of evidence expand the knowledge about its role in immune regulation. ${ }^{19-21}$ Animal and clinical studies have demonstrated altered Cyr61 expression in diseased kidneys. ${ }^{22-24}$ We have shown that upregulated Cyr61 expression mediates proinflammatory effects in animal models of obstructive renal fibrosis and ischaemia-reperfusion kidney injury. ${ }^{20} 25$ In a pilot clinical observation, urinary Cyr61 excretion was significantly increased in patients with dialysis-requiring acute kidney injury. ${ }^{25}$ These results indicate that urinary Cyr61 may be a potential biomarker reflecting active and/or progressive kidney injury.

We hypothesised that renal Cyr61 expression is enhanced in patients with advanced CKD and is associated with kidney function deterioration. To test this hypothesis, we conducted this clinical study to examine urinary Cyr61 excretion in patients with various stages of CKD. Special attention was given to the associations between urinary Cyr61 excretion and subsequent kidney function changes.

\section{MATERIALS AND METHODS \\ Patients}

This observational cohort study was conducted in the National Taiwan University Hospital, a tertiary hospital in Taiwan. From February to October 2014, adult patients with stages 1-5 CKD followed in the nephrology outpatient clinics (C-FL, C-CF, Y-MC, M-SW and T-JT) were recruited. CKD was defined and staged by the GFR category of the 2012 Kidney Disease Improving Global Outcome criteria. ${ }^{2}$ Exclusion criteria included patients younger than 20 or older than 80 years, pregnant women and patients with ESRD under maintenance haemodialysis, peritoneal dialysis or receiving kidney transplantation.

Baseline demographic and clinical data were collected from medical records. Comorbidities of the subjects were recorded according to the diagnosis and medication used at study entry. Each patient's primary care nephrologist ordered laboratory testing as standard clinical practice. ${ }^{24}$ These laboratory measurements were carried out by the Department of Laboratory Medicine, National Taiwan University Hospital. Serum creatinine levels were measured by the AU-5800 analyzer (Beckman Coulter, Brea, California, USA), and the determinations were calibrated to an isotope dilution mass spectrometry (IDMS) reference method. We used the IDMS-traceable Modification of Diet in Renal Disease equation [175×serum creatinine ${ }^{-1.154} \times$ Age ${ }^{-0.203} \times 0.742$ (if woman)] to calculate eGFR. ${ }^{26}$ The most recent results before the date of enrolment were used.

\section{Urinary protein and Cyr61 excretion}

We collected $5 \mathrm{~mL}$ of random spot urine on the day of enrolment. The samples were centrifuged at $2000 \mathrm{~g}$ for 5 $\mathrm{min}$, and the supernatants were stored in equal volumes at $-80^{\circ} \mathrm{C}$ before measurements. Urinary total protein and creatinine concentrations were measured by the AU-5800 analyzer. Urinary Cyr61 concentrations were measured by the use of an ELISA kit (EIA-5108, DRG, Marburg, Germany) according to the manufacturer's instructions ${ }^{25}$ Measured urine Cyr61 concentrations below the limit-ofdetection (LOD) of the assay $(14.9 \mathrm{pg} / \mathrm{mL})$ were substituted to one-half the LOD, that is, $7.45 \mathrm{pg} / \mathrm{mL}^{27}{ }^{28}$ All measurements were made in duplicate and in a blinded fashion. The measured urinary total protein and Cyr61 values were normalised to the urinary creatinine concentration and are expressed as the urine protein-to-creatinine ratio (UPCR, g/g creatinine) or urine Cyr61-to-creatinine ratio (UCyr61CR, ng/g creatinine).

\section{Kidney function changes}

This cohort was prospectively observed for eGFR change without a mandate for study-specific visits or laboratory tests. The observation period of each patient was defined to start immediately after enrolment and lasted for 1 year or until ESRD or death, whichever came first. ESRD was diagnosed if the patient initiated long-term renal replacement therapy.

Patients were routinely followed at the nephrology outpatient clinic with standard care. ${ }^{2}$ In general, the participants returned to the clinic and received routine laboratory tests every 1-3 months, according to the judgement of their primary care nephrologists. All available laboratory data during the follow-up were recorded. The serum creatinine levels, which were measured on the date closest to the 3 months or 1 year after enrolment, were obtained to calculate eGFR at that time point. The eGFR decline rate was calculated by the following equation:

eGFR decline rate $\left(\mathrm{ml} / \mathrm{min} / 1.73 \mathrm{~m}^{2} /\right.$ year $)=$

(baseline eGFR - eGFR at approximately 3 months or 1 year after enrolment) ( $\left.\frac{\text { exact days between the two serum creatinine measurements }}{365.25}\right)$

For each participant, we calculated 3-month and 1-year eGFR decline rates separately. Rapid kidney function decline was defined as either having an eGFR decline 
rate $\geq 4 \mathrm{~mL} / \mathrm{min} / 1.73 \mathrm{~m}^{2} /$ year $^{2} 2930$ or developing ESRD during the specific period.

\section{Sample size estimation}

In the literature, there is no clinical report regarding urinary Cyr61 in patients with CKD. In a pilot observation, ${ }^{25}$ the $\log$ (UCyr61CR ng/g) was $3.64 \pm 0.88$ in patients with dialysis-requiring acute kidney injury and $1.93 \pm 0.84$ in controls. We proposed that patients with CKD would have a lower mean level of $\log$ (UCyr61CR) than dialysisrequiring acute kidney injury. Assuming that the mean $\log$ (UCyr61CR) was 2.6 and 1.93 in patients with CKD with and without rapid kidney function decline, respectively, an SD of 1.0, a type 1 error of $5 \%$, and a desired power of $85 \%$, we calculated a minimal sample size of 41 participants in each group.

\section{Statistical analyses}

Baseline categorical variables are presented as number and percentage, while continuous variables are presented as mean and SD or median with IQR. Characteristics were compared between the groups by the $\chi^{2}$ test, Fisher's exact test, one-way analysis of variance test, t-tests, Kruskal-Wallis test or Mann-Whitney U test, as indicated. Age, sex, body weight, hypertension, haemoglobin, baseline eGFR and proteinuria were selected in the stepwise multiple linear regression model for urinary Cyr61 excretion. In addition, logistic regression was employed to model the OR of rapid kidney function decline during the subsequent 3 months or 1 year in separate analyses. Patients lost to follow-up or who did not have serum creatinine measurements at the corresponding time points were excluded from these analyses. Unadjusted ORs were further adjusted in regression models by adding the covariates age, sex, diabetes, hypertension, hyperlipidaemia, CKD aetiology, body weight, haemoglobin, baseline eGFR and proteinuria. Statistical tests for multicollinearity by checking variance inflation factors were used to assess problems within the regression model. All of the tests were two tailed, with significance defined by $p$ values of less than 0.05 . The statistical packages STATA V.10.0 and R V.3.5.3 were used for the statistical analyses.

\section{Patient and public involvement}

Patients and the public were not involved in the design, or conduct, or reporting, or dissemination plans of this study.

\section{RESULTS}

\section{Patient characteristics}

A total of 138 outpatients with documented CKD were enrolled. Their baseline demographic and clinical characteristics are summarised in table 1, online supplemental table 1 and online supplemental figure 1 . Their mean age was $60.46 \pm 13.16$ years; 51 of them were women $(37.0 \%) ; 115$ (83.3\%) had hypertension; and 53 (38.4\%) had diabetes. At the beginning of the study, $12(8.7 \%)$ patients were at CKD stage 1, $32(23.2 \%)$ at stage 2, 18
$(13.0 \%)$ at stage $3 a, 18(13.0 \%)$ at stage 3b, $29(21.0 \%)$ at stage 4 and $29(21.0 \%)$ at stage 5 (online supplemental figure 2).

\section{Urinary Cyr61 excretion}

Urinary Cyr61 concentrations were below the LOD (14.9 $\mathrm{pg} / \mathrm{mL})$ in $47(75.8 \%)$ of the 62 patients with baseline eGFR $\geq 45 \mathrm{~mL} / \mathrm{min} / 1.73 \mathrm{~m}^{2}$, as well as in $23(30.3 \%)$ of the 76 patients with baseline eGFR $<45 \mathrm{~mL} / \mathrm{min} / 1.73$ $\mathrm{m}^{2}$. The urinary Cyr61 concentrations of these 70 samples were substituted to $7.45 \mathrm{pg} / \mathrm{mL} \cdot{ }^{27}{ }^{28}$ As shown in figure 1A,B, the distributions of UCyr61CR and UPCR were highly skewed. Patients with stage 4 or 5 CKD had significantly higher UCyr61CR than patients with stages1-3a CKD (Kruskal-Wallis test, $\mathrm{p}<0.001$; each pairwise comparison with Bonferroni correction was shown in figure 1C). UCyr61CR in patients with stage 4 CKD was comparable to that in patients with stage $3 \mathrm{~b}$ or $5 \mathrm{CKD}$ (both $\mathrm{p}=1$ with Bonferroni correction).

Because the UPCR and UCyr61CR were highly skewed, they were log-transformed to achieve approximate normality (online supplemental figure 3). Unadjusted associations of demographic characteristics with urinary Cyr61 were shown in online supplemental table 2 and online supplemental figure 4. $\log$ (UCyr61CR) was positively associated with age and $\log$ (UPCR), while it was negatively associated with eGFR, haemoglobin and serum calcium. There was no difference in UCyr61CR between groups in terms of sex, diabetes, hypertension, hyperlipidaemia and CKD aetiology. Multivariable stepwise regression analysis revealed that $\log$ (UCyr61CR) was independently associated with eGFR ( $\beta$-coefficient $=-0.016$, $\mathrm{p}<0.001), \log (\mathrm{UPCR}) \quad(\beta$-coefficient $=0.538, \mathrm{p}<0.001)$ and hypertension $(\beta$-coefficient $=-0.494, \mathrm{p}=0.007)$.

Figure 1D reveals that UCyr61CR was low in most patients with baseline eGFR $\geq 45 \mathrm{~mL} / \mathrm{min} / 1.73 \mathrm{~m}^{2}$. Therefore, we investigated a subset of 76 patients with baseline eGFR $<45 \mathrm{~mL} / \mathrm{min} / 1.73 \mathrm{~m}^{2}$ (table 1 ). Most of the associations of demographic characteristics with UCyr61CR were similar to those observed in the entire cohort (online supplemental table 2). Multivariable stepwise regression analysis revealed that $\log (\mathrm{UCyr} 61 \mathrm{CR})$ in this subset was independently associated with eGFR ( $\beta$-coefficient $=-0.032, \mathrm{p}=0.002), \log (\mathrm{UPCR}) \quad(\beta$-coefficient $=0.82$, $\mathrm{p}<0.001)$, hypertension $(\beta$-coefficient $=-1.16, \mathrm{p}=0.001)$ and age $(\beta$-coefficient $=0.025, \mathrm{p}=0.002)$.

\section{Kidney function decline}

Among the 138 included patients, 107 (77.5\%) had serum creatinine measurements at baseline, 3 months and 1 year. Others had only one or two measurements at these time points (online supplemental figure 5). Five patients developed ESRD, while three patients were lost to follow-up during the 1-year follow-up. No participant died within 1 year after enrolment.

We analysed the eGFR changes in those who had serum creatinine measurements at all of the three time points or developed ESRD during the follow-up ( $\mathrm{n}=112$, online 
Table 1 Demographics and clinical characteristics of the patients

\begin{tabular}{|c|c|c|}
\hline Characteristic & All patients $(n=138)$ & Subset with eGFR $<45 \mathrm{~mL} / \mathrm{min} / 1.73 \mathrm{~m}^{2}(\mathrm{n}=76)$ \\
\hline Age (years) & $60.46 \pm 13.16$ & $63.14 \pm 12.71$ \\
\hline Sex, women (\%) & $51(37.0)$ & $26(34.2)$ \\
\hline Body height $(\mathrm{cm})$ & $163.06 \pm 8.49$ & $163.1 \pm 8.97$ \\
\hline Body weight (kg) & $65.61 \pm 12.95$ & $64.25 \pm 13.37$ \\
\hline Diabetes (\%) & $53(38.4)$ & $33(43.4)$ \\
\hline Hypertension (\%) & $115(83.3)$ & $69(90.8)$ \\
\hline Hyperlipidaemia (\%) & $89(64.5)$ & $48(63.2)$ \\
\hline \multicolumn{3}{|l|}{ Aetiology (\%) } \\
\hline Diabetic nephropathy & $40(29.0)$ & $26(34.2)$ \\
\hline CGN & $51(37.0)$ & $21(27.6)$ \\
\hline Hypertensive & $20(14.5)$ & $13(17.1)$ \\
\hline CTIN & $6(4.3)$ & $5(6.6)$ \\
\hline PCKD & $7(5.1)$ & $5(6.6)$ \\
\hline Others & $14(10.1)$ & $6(7.9)$ \\
\hline eGFR $\left(\mathrm{mL} / \mathrm{min} / 1.73 \mathrm{~m}^{2}\right)$ & $44.9 \pm 30.96$ & $20.85 \pm 10.62$ \\
\hline Urinary Cyr61 (pg/mL)* & $7.45(7.45,622.59)$ & $461.81(7.45,2547.49)$ \\
\hline UCyr61CR (ng/g) * & $49.41(18.84,943.21)$ & $695.21(22.09,4035.7)$ \\
\hline Log(UCyr61CR) & $1.92 \pm 1.12$ & $2.54 \pm 1.1$ \\
\hline UPCR $(g / g)^{*}$ & $0.36(0.09,1.03)$ & $0.76(0.33,1.83)$ \\
\hline Log(UPCR) & $-0.48 \pm 0.7$ & $-0.19 \pm 0.62$ \\
\hline Haemoglobin (g/L) & $126.85 \pm 22.19$ & $114.55 \pm 18.79$ \\
\hline White cell count $(x 10 \wedge 9 / L)$ & $7.02 \pm 2.02$ & $6.94 \pm 1.91$ \\
\hline Fasting glucose $(\mathrm{mg} / \mathrm{dL}) \dagger$ & $113.9 \pm 32.34$ & $121.19 \pm 38.28$ \\
\hline Albumin $(\mathrm{g} / \mathrm{dL}) \dagger$ & $4.19 \pm 0.39$ & $4.15 \pm 0.41$ \\
\hline $\mathrm{K}(\mathrm{mmol} / \mathrm{L}) \dagger$ & $4.51 \pm 0.6$ & $4.62 \pm 0.64$ \\
\hline $\mathrm{Ca}(\mathrm{mmol} / \mathrm{L}) \dagger$ & $2.26 \pm 0.13$ & $2.24 \pm 0.12$ \\
\hline $\mathrm{P}(\mathrm{mg} / \mathrm{dL}) \dagger$ & $3.82 \pm 0.89$ & $3.91 \pm 0.89$ \\
\hline Triglyceride (mg/dL)† & $189.65 \pm 146.61$ & $193.95 \pm 119.11$ \\
\hline Total cholesterol (mg/dL)† & $178.03 \pm 34.61$ & $178.02 \pm 36.03$ \\
\hline
\end{tabular}

The data are presented as the means (SD) or number (\%) unless otherwise specified.

${ }^{*}$ Presented as median (25th/75th percentile).

†Not all subjects had these laboratory values. Numbers with results of the specified tests (in all patients and subset with eGFR $<45 \mathrm{~mL} /$ min/1.73 $\mathrm{m}^{2}$, respectively) were: fasting glucose $(n=80,37)$; albumin $(n=79,60) ; K(n=92,66) ; C a(n=58,51) ; P(n=60,52) ;$ triglyceride $(n=63$, $38)$; total cholesterol $(n=77,42)$. For these values, analyses were only done on variables present with missing observations ignored. Variables with missing data were not included in the multivariable analyses.

CGN, chronic glomerulonephritis; CTIN, chronic tubulointerstitial nephritis; Cyr61, cysteine-rich protein 61; eGFR, estimated glomerular filtration rate; PCKD, polycystic kidney disease; UCyr61CR, urine Cyr61-to-creatinine ratio; UPCR, urine protein-to-creatinine ratio.

supplemental figure 5A). At 3 months after enrolment, 47 $(42.0 \%)$ of the 112 patients showed a rapid kidney function decline during the 3-month period, with a median eGFR decline rate 11.79 (IQR 6.63-25.96) $\mathrm{ml} / \mathrm{min} / 1.73$ $\mathrm{m}^{2} /$ year. As shown in figure 2A, baseline $\log$ (UCyr61CR) was significantly higher in patients with rapid decline during the subsequent 3 months $(\mathrm{p}<0.001)$. Unadjusted logistic regression analysis showed that $\log$ (UCyr61CR) was associated with rapid kidney function decline (OR $1.78,95 \%$ CI 1.25 to $2.54, \mathrm{p}=0.001$ ). After adjustment for age, sex, body weight, diabetes, hypertension, hyperlipidaemia, CKD aetiology, haemoglobin, baseline eGFR and proteinuria, $\log$ (UCyr61CR) remained associated with rapid kidney function decline in the subsequent 3 months (adjusted OR 2.29, 95\% CI 1.27 to 4.15 , $\mathrm{p}=0.006$, table 2 ).

Among the 112 patients who were analysed for kidney function change, 38 (33.9\%) experienced rapid kidney function decline at 1 year after enrolment, with a median eGFR decline rate 7.98 (IQR 5.85-10.41) $\mathrm{ml} / \mathrm{min} / 1.73$ $\mathrm{m}^{2} /$ year. $\log (\mathrm{UCy} 61 \mathrm{CR})$ was comparable between groups with and without rapid decline (figure 2B, $\mathrm{p}=0.15$ ). 

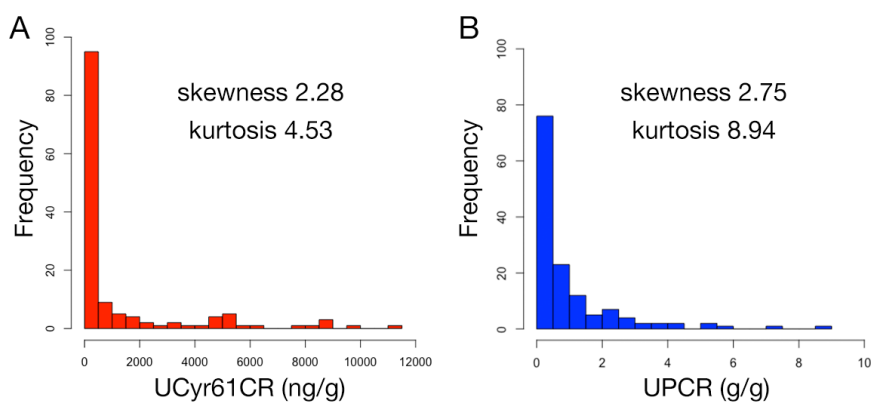

C

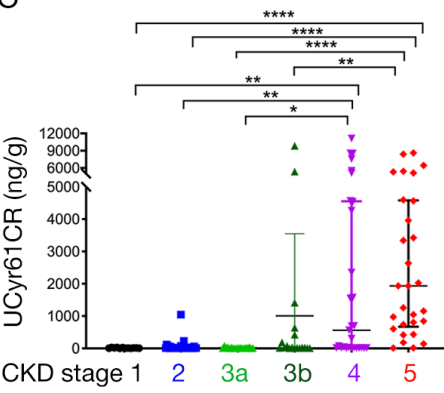

D

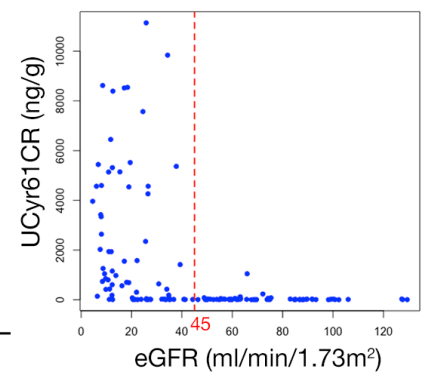

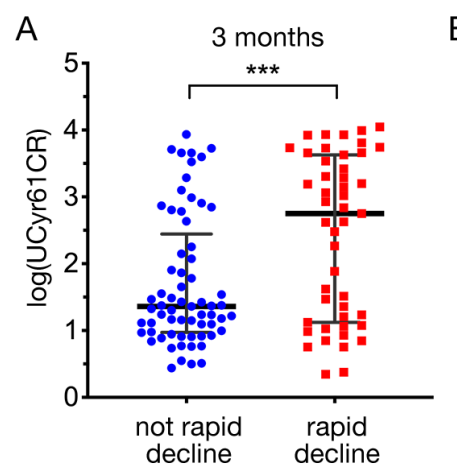

C

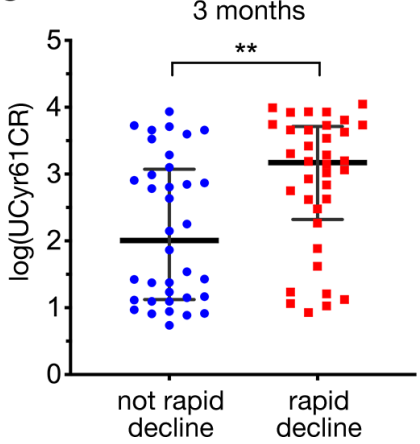

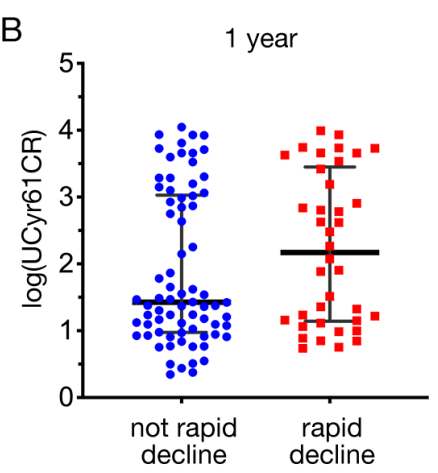

D

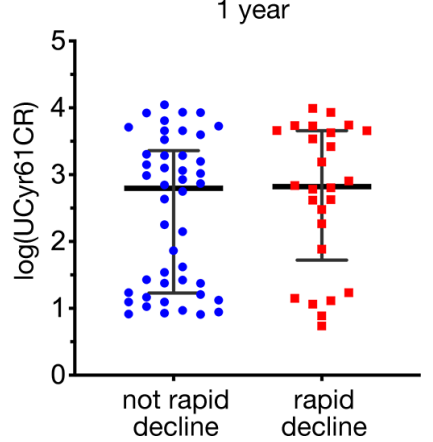

Figure 1 Urinary Cyr61 and total protein levels. (A, distributions of $(A)$ the urine Cyr61-to-creatinine ratio (UCyr61CR) and (B) the urine protein-to-creatinine ratio (UPCR) in patients with chronic kidney disease (CKD). (C) UCyr61CR levels (with lines at the median, first quartile and third quartile) in patients with stage 4 or 5 CKD are significantly higher than those in patients with stages 1, 2 or 3 a CKD. Kruskal-Wallis test, $p<0.001$; Mann-Whitney $U$ test with Bonferroni correction: ${ }^{\star} \mathrm{p}<0.05$; ${ }^{\star *} \mathrm{p}<0.01$; ${ }^{* \star *} \mathrm{p}<0.0001$. (D) Scatter plot of UCyr61CR and baseline estimated glomerular filtration rate (eGFR). Cyr61, cysteine-rich protein 61.

Baseline urinary Cyr61 excretion was not associated with rapid kidney function decline in the subsequent 1 year (table 2). Encountering rapid kidney function decline at 3 months was associated with the risk of rapid kidney function decline at 1 year (OR 4.55, 95\% CI 1.97 to 10.48 , $\mathrm{p}<0.001)$.

In the subset of patients whose baseline eGFR $<45 \mathrm{~mL} /$ $\min / 1.73 \mathrm{~m}^{2}, 72$ patients had serum creatinine measurements at the three time points or developed ESRD during the follow-up. There were $36(50 \%)$ patients experienced rapid kidney function decline in the subsequent 3 months. Baseline $\log$ (UCyr61CR) was significantly higher in patients with rapid decline in the subsequent 3 months (figure 2C, $\mathrm{p}=0.005$ ). At 1 year after enrolment, $26(36 \%)$ had rapid kidney function decline during this 1-year period. Baseline $\log (\mathrm{UCyr} 61 \mathrm{CR})$ values were not different between the groups (figure 2D, $\mathrm{p}=0.35$ ). In accordance with the results from the entire cohort, baseline $\log$ (UCyr61CR) had increased odds for rapid eGFR decline in the subsequent 3 months (table 2, figure $3 \mathrm{~A}$ ).

We then examine whether measuring urinary Cyr61 excretion has clinical relevance for short-term kidney function change. Based on the previous reports in the literature, age, sex, baseline eGFR and $\log$ (UPCR) were included in a base clinical model. ${ }^{31}$ In our cohort, this

Figure 2 Associations of urinary Cyr61 and subsequent rapid kidney function decline. (A, B) Comparisons of baseline log(UCyr61CR) in patients grouped by their kidney function decline rate in the subsequent (A) 3 month and (B) 1-year follow-up period. Rapid kidney function decline was defined as an eGFR decline rate $\geq 4 \mathrm{~mL} / \mathrm{min} / 1.73 \mathrm{~m}^{2} /$ year or developing end-stage renal disease during the specific time period. (C, D) In a subset of patients with baseline eGFR $<45$ $\mathrm{mL} / \mathrm{min} / 1.73 \mathrm{~m}^{2}$, comparisons of baseline log(UCyr61CR) in patients grouped by their kidney function decline rate in the subsequent (C) 3 months and (D) 1-year follow-up period. Lines at median and first and third quartiles. ${ }^{* *} \mathrm{p}<0.01$; ${ }^{\star \star *} \mathrm{p}<0.001$. Cyr61, cysteine-rich protein 61 ; eGFR, estimated glomerular filtration rate; UCyr61CR, urine Cyr61-tocreatinine ratio.

model exhibited a suboptimal performance to predict rapid kidney function decline during the 3-month period, with the area under the receiver operating characteristic curve (AUROC) $66.84 \%$. Using a cut-off of $\log$ (UCyr61CR) 2.27 (ie, UCyr61CR $186 \mathrm{ng} / \mathrm{g}$ ) or higher for predicting rapid 3-month kidney function decline demonstrated the highest Youden's index: the sensitivity was $59.57 \%$, specificity was $75.38 \%$, positive predictive value was $63.64 \%$, negative predictive value was $72.06 \%$ and the AUROC was $67.48 \%$. Adding the UCyr61CR $\geq 186$ $\mathrm{ng} / \mathrm{g}$ into the base clinical model improve the AUROC to $73.36 \%$, indicating good discrimination (figure $3 \mathrm{~B}$ ).

\section{DISCUSSION}

Many clinical factors, such as age, sex, race, blood pressure, serum albumin, haemoglobin and urinary protein excretion, have been reported to be associated with the deterioration of kidney function. ${ }^{31-33}$ Blood and/or urinary levels of neutrophil gelatinase-associated lipocalin, kidney injury molecule-1, cystatin $\mathrm{C}$, dimethylarginines and 
Table 2 Association of baseline log(UCyr61CR) with rapid kidney function decline

\begin{tabular}{|c|c|c|c|c|}
\hline & \multicolumn{2}{|c|}{$\begin{array}{l}\text { Patients analysed for kidney function } \\
\text { decline }(n=112)\end{array}$} & \multicolumn{2}{|c|}{$\begin{array}{l}\text { Subset with baseline eGFR }<45 \mathrm{~mL} / \\
\min / 1.73 \mathrm{~m}^{2}(\mathrm{n}=72)\end{array}$} \\
\hline & OR (95\% Cl) & $P$ value & OR $(95 \% \mathrm{Cl})$ & $P$ value \\
\hline \multicolumn{5}{|l|}{ 3-month follow-up } \\
\hline Unadjusted & $1.78(1.25$ to 2.54$)$ & 0.001 & $1.92(1.2$ to 3.07$)$ & 0.006 \\
\hline Multivariable adjusted model 1 & 2.16 (1.24 to 3.78$)$ & 0.007 & 3.18 (1.39 to 7.29$)$ & 0.006 \\
\hline Multivariable adjusted model 2 & 2.12 (1.21 to 3.73$)$ & 0.009 & 3.29 (1.41 to 7.64$)$ & 0.006 \\
\hline Multivariable adjusted model 3 & 2.29 (1.27 to 4.15$)$ & 0.006 & 3.22 (1.32 to 7.88$)$ & 0.01 \\
\hline \multicolumn{5}{|l|}{ 1-year follow-up } \\
\hline Unadjusted & $1.29(0.91$ to 1.83$)$ & 0.146 & 1.24 (0.79 to 1.96$)$ & 0.345 \\
\hline Multivariable adjusted model 1 & $1.26(0.73$ to 2.2$)$ & 0.407 & 1.47 (0.69 to 3.14$)$ & 0.317 \\
\hline Multivariable adjusted model 2 & 1.34 (0.75 to 2.39$)$ & 0.327 & 1.35 (0.63 to 2.88$)$ & 0.435 \\
\hline Multivariable adjusted model 3 & $1.3(0.7$ to 2.4$)$ & 0.406 & 0.99 (0.42 to 2.37$)$ & 0.986 \\
\hline
\end{tabular}

Rapid kidney function decline was defined as an eGFR decline rate $\geq 4 \mathrm{~mL} / \mathrm{min} / 1.73 \mathrm{~m}^{2} /$ year or developing end-stage renal disease during the specific follow-up period.

Multivariable adjusted model 1: adjusted for baseline eGFR, log urinary protein to creatinine ratio, hypertension, age.

Multivariable adjusted model 2: adjusted for model 1 variables+body wt and haemoglobin.

Multivariable adjusted model 3: adjusted for model 2 variables+sex, diabetes, hyperlipidaemia and CKD aetiology.

CKD, chronic kidney disease; eGFR, estimated glomerular filtration rate; UCyr61CR, urine cysteine-rich protein 61-to-creatinine ratio.

fibroblast growth factor-23 were also independent predictors of disease severity and progression of CKD. ${ }^{34-36}$ Integrating clinical factors with biomarkers has the potential to predict kidney disease trajectory and to guide prompt interventions more efficiently. ${ }^{3738}$ In this study, we found that urinary Cyr61 levels were very low in most patients with stages 1, 2 and 3a CKD. In contrast, urinary Cyr61
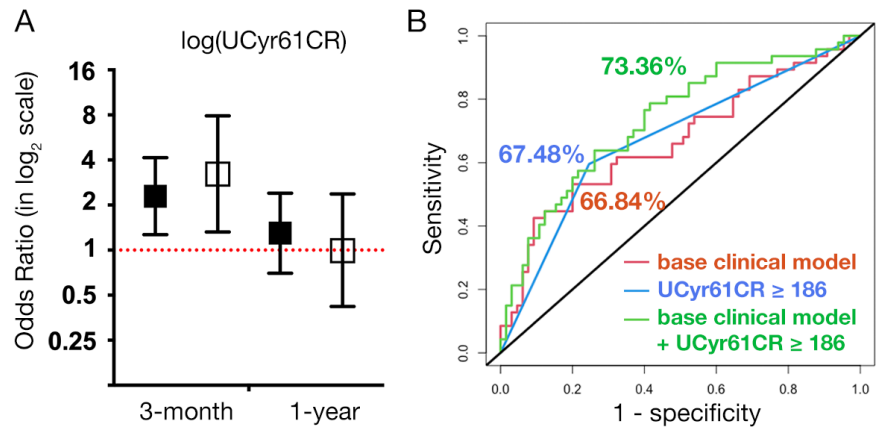

Figure 3 Urinary Cyr61 and protein as predictors of rapid kidney function decline in patients with CKD. (A) Adjusted ORs and $95 \%$ Cls of baseline log(UCyr61CR) for rapid kidney function decline during different time periods in the entire cohort (solid boxes) and in a subset of patients with baseline EGFR $<45 \mathrm{~mL} / \mathrm{min} / 1.73 \mathrm{~m}^{2}$ (hollow boxes). Multivariable logistic analyses included age, sex, diabetes, hypertension, hyperlipidaemia, CKD aetiology, body weight, haemoglobin, baseline eGFR, log(UCyr61CR) and log(UPCR). (B) The area under the receiver operating characteristic curve (AUROC) for predicting rapid kidney function decline at 3 months followup by the base clinical model (red), UCyr61CR $\geq 186 \mathrm{ng} / \mathrm{g}$ (blue), and an integrated model of adding log(UCyr61CR) to the base clinical model (green). Cyr61, cysteine-rich protein 61; eGFR, estimated glomerular filtration rate; UCyr61CR, urine Cyr61-to-creatinine ratio; UPCR, urine protein-tocreatinine ratio. increased significantly in patients with stages 4 and 5 CKD. Multivariable analysis showed that $\log$ (UCyr61CR) was negatively associated with eGFR, independent of age, sex, body weight, haemoglobin level and the severity of proteinuria. Therefore, although urinary Cyr61 excretion is not a sensitive biomarker for detecting early CKD, it is an indicator suggesting advanced kidney disease status.

There are some possible explanations for this association. First, animal studies have shown that upregulation of renal tubular Cyr61 and granulocyte-macrophage colonystimulating factor contributes to renal inflammation and fibrosis through monocyte chemoattractant protein-1 induction. ${ }^{10} 1420$ Transcriptional profiling of progressive fibrotic murine kidneys revealed the increased expression of a variety of proinflammatory gene modules. ${ }^{13}$ LebherzEichinger et al also demonstrated different patterns of urinary chemokine excretion in different stages of CKD. ${ }^{9}$ In other reports, urinary levels of inflammatory proteins have been elevated in accordance with disease activity in patients with diabetic nephropathy and lupus nephritis. ${ }^{15}{ }^{39}$ As such, elevated urinary Cyr61 expression may reflect persistent inflammatory processes in kidneys.

Second, there was a positive correlation of urinary total protein with Cyr61 excretion (online supplemental figure $4 \mathrm{C}$ ). It is possible that the increased urinary Cyr61 excretion came from the overflow of proteinuria. However, our multivariable regression analysis showed that the association of $\log$ (UCyr61CR) and eGFR remained significant after adjusting for $\log (\mathrm{UPCR})$. This suggests a specific role of urinary Cyr61 excretion, independent of the extent of proteinuria. Third, advanced kidney failure may lead to Cyr61 accumulation in the body. Since blood Cyr61 levels were not measured in this study, we could not examine this 
hypothesis. If this scenario is true, renal clearance of Cyr61 should decrease as kidney function declines. This contradicts the negative correlation of $\log$ (UCyr61CR) and eGFR we found (online supplemental figure 4B). As a result, the elevated urinary Cyr61 excretion in advanced CKD is not merely due to the worsened eGFR.

The association between urinary Cyr61 excretion and rapid kidney function decline in the subsequent 3-month period deserves more discussion. A recent single cell RNA sequencing study suggested Cyr61 is one of the chemotactic signalings upregulated in failed repair renal tubules during progressive kidney disease. ${ }^{14}$ Our previous animal study also demonstrated that blocking Cyr61 ameliorates renal inflammation, interstitial fibrosis and capillary rarefaction after ischaemic kidney injury. ${ }^{25}$ It may be possible that elevated urinary Cyr61 reflects active intrarenal inflammation along with ongoing kidney function deterioration, no matter whether it is consequential or causative. Since the current study did not measure other inflammatory cytokines and did not interfere with Cyr61, the causal relationship remains to be investigated by mechanistic studies.

As shown in figure 3B, UCyr61CR measurement could improve the discriminative performance of clinical models to predict rapid kidney function decline in the subsequent 3-month period. However, it was not associated with rapid kidney function decline in the whole 1-year observation period (figure 3A). Along with different disease activities manifesting at different time points and the response to treatments, urinary Cyr61 excretion may change with time. Therefore, urinary Cyr61 measurement at a single time point can predict subsequent short-term kidney function decline, but not long-term deleterious renal outcomes. Since urinary Cry61 measurement is non-invasive, serial monitoring this biomarker may be more beneficial to guide clinical patient care.

Our study had certain limitations. First, nondetectable urine Cyr61 values were substituted to one-half the LOD of the assay. We also performed supplementary analyses by using other substitution methods and demonstrated very similar results (online supplemental figure 6). Although the LOD/2 substitution method appears to give sufficiently accurate results when the data are highly skewed, ${ }^{27}$ it leads to a negative bias for variability and does not estimate the mean well. Second, this observational study was conducted in a single hospital, and the sample size was relatively small. We were unable to fully adjust for all potential confounders, such as diet, fluids status and medication that can contribute to CKD progression. The association we identified lacked generalisability and did not imply causality. Third, although most patients were enrolled in the outpatient setting without frequent recent nephrology appointments (online supplemental figure 1), the entry points of patients to the study are not the same. Patients who had acute kidney injury prior to the enrolment or encountered subsequent acute event might trigger the deterioration of kidney function, rather than CKD progression itself. Fourth, we did not prespecify a schedule of laboratory tests during the follow-up. It has been shown that patients may have nonlinear trajectories of eGFR change. ${ }^{29}$ Using only two serum creatinine measurements to estimate the slopes of eGFR changes in the current study may be misleading. The accuracy of assessing kidney function trajectory can be improved by increasing the number of serum creatinine measurements and/or using the mixed effect model. ${ }^{2}{ }^{15} 40$ Besides, we measured the Cyr61 concentration of random urine samples. This did not take into account the possible diurnal variation in urinary Cyr61 excretion. Furthermore, we did not measure serial urinary Cyry61 levels longitudinally. We cannot comment on the change in urinary Cyr61 as a function of eGFR change or its value in predicting the response to therapies.

In conclusion, urinary Cyr61 excretion was significantly increased in patients with advanced CKD. The extent of urinary Cyr61 was associated with rapid shortterm kidney function decline. Non-invasive urinary Cyr61 measurement can be clinically valuable for monitoring short-term kidney function trajectory and can help guide treatment planning earlier in patients with CKD.

\section{Author affiliations}

${ }^{1}$ Department of Internal Medicine, National Taiwan University Hospital and National Taiwan University College of Medicine, Taipei, Taiwan

${ }^{2}$ Jia Hong Clinic, Tainan, Taiwan

${ }^{3}$ Department of Family Medicine, Mackay Memorial Hospital, Taipei, Taiwan ${ }^{4}$ Department of Internal Medicine, Far Eastern Memorial Hospital, New Taipei City, Taiwan

${ }^{5}$ School of Medicine, College of Medicine, National Yang Ming Chiao Tung University, Taipei, Taiwan

${ }^{6}$ Institute of Epidemiology and Preventive Medicine, College of Public Health, National Taiwan University, Taipei, Taiwan

${ }^{7}$ Department of Emergency Medicine, National Taiwan University Hospital and National Taiwan University College of Medicine, Taipei, Taiwan

${ }^{8}$ Department of Geriatrics and Gerontology, National Taiwan University Hospital and National Taiwan University College of Medicine, Taipei, Taiwan

Acknowledgements We thank Miss Shwu-Juant Tsai and Dr. Kuo-Tong Huang for their assistant. We also thank the staff of the 7th Core Labs, Department of Medical Research at National Taiwan University Hospital for technical support.

Contributors Concept and design: C-FL, C-CF and T-JT. Acquisition, analysis and interpretation of data: all authors. Drafting of the manuscript: $\mathrm{C}-\mathrm{FL}$ and $\mathrm{H}-\mathrm{YW}$. Critical revision of the manuscript for important intellectual content: J-JW, Y-CT, C-YH, Y-MC, M-SW and T-JT. Statistical analysis: C-FL and H-YW. Obtained funding and study supervision: C-FL, H-YW and T-JT. All authors have seen the final version of manuscript, endorse the data, and approve the conclusions. All authors agree to be accountable for all aspects of the work in ensuring that questions related to the accuracy or integrity of any part of the work are appropriately investigated and resolved.

Funding This work was supported by the Far Eastern Memorial Hospital-National Taiwan University Hospital Joint Research Programme (104-FTN10), Ministry of Science and Technology, Taiwan (MOST 104-2314-B-002-165-MY3, MOST 107-2314-B-002-177-MY3), National Health Research Institutes, Taiwan (NHRIEX110-11026PI), Mrs Hsiu-Chin Lee Kidney Research Fund, and National Taiwan University (Aim for Top University Programme 102R-7620 R-7620).

Competing interests None declared. 
Patient consent for publication Not applicable.

Ethics approval This study was approved by the Institutional Review Board of the National Taiwan University Hospital, Taipei, Taiwan (201309015RIN). All participants signed a written informed consent form before inclusion in the study.

Provenance and peer review Not commissioned; externally peer reviewed.

Data availability statement Data are available on reasonable request.

Supplemental material This content has been supplied by the author(s). It has not been vetted by BMJ Publishing Group Limited (BMJ) and may not have been peer-reviewed. Any opinions or recommendations discussed are solely those of the author(s) and are not endorsed by BMJ. BMJ disclaims all liability and responsibility arising from any reliance placed on the content. Where the content includes any translated material, BMJ does not warrant the accuracy and reliability of the translations (including but not limited to local regulations, clinical guidelines, terminology, drug names and drug dosages), and is not responsible for any error and/or omissions arising from translation and adaptation or otherwise.

Open access This is an open access article distributed in accordance with the Creative Commons Attribution Non Commercial (CC BY-NC 4.0) license, which permits others to distribute, remix, adapt, build upon this work non-commercially, and license their derivative works on different terms, provided the original work is properly cited, appropriate credit is given, any changes made indicated, and the use is non-commercial. See: http://creativecommons.org/licenses/by-nc/4.0/.

\section{ORCID iDs}

Chun-Fu Lai http://orcid.org/0000-0003-2109-1522

Cheng-Chung Fang http://orcid.org/0000-0001-5386-6674

\section{REFERENCES}

1 United States Renal Data System. 2019 USRDS annual data report: epidemiology of kidney disease in the United States. Bethesda, MD: National Institutes of Health, National Institute of Diabetes and Digestive and Kidney Diseases, 2019.

2 Kidney disease: improving global outcomes (KDIGO) CKD work group. Chapter 2: definition, identification, and prediction of CKD progression. Kidney Int Suppl 2013;3:63-72.

3 Chen PM, Lai TS, Chen PY, et al. Multidisciplinary care program for advanced chronic kidney disease: reduces renal replacement and medical costs. Am J Med 2015;128:68-76.

4 National Health Research Institutes, Taiwan. Taiwan chronic kidney disease clinical guidelines. Zhunan, Miaoli County, Taiwan: National Health Research Institutes, Taiwan, 2015.

5 Lin C-M, Yang M-C, Hwang S-J, et al. Progression of stages 3b-5 chronic kidney disease--preliminary results of Taiwan national preESRD disease management program in Southern Taiwan. J Formos Med Assoc 2013;112:773-82.

6 Glassock RJ, Warnock DG, Delanaye P. The global burden of chronic kidney disease: estimates, variability and pitfalls. Nat Rev Nephrol 2017:13:104-14.

7 Humphreys BD. Mechanisms of renal fibrosis. Annu Rev Physiol 2018;80:309-26.

8 Declèves A-E, Sharma K. Novel targets of antifibrotic and antiinflammatory treatment in CKD. Nat Rev Nephrol 2014;10:257-67.

9 Lebherz-Eichinger D, Klaus DA, Reiter T, et al. Increased chemokine excretion in patients suffering from chronic kidney disease. Trans/ Res 2014;164:433-43.

10 Xu L, Sharkey D, Cantley LG. Tubular GM-CSF promotes late MCP-1/CCR2-Mediated fibrosis and inflammation after ischemia/ reperfusion injury. J Am Soc Nephrol 2019;30:1825-40.

11 Beckerman P, Qiu C, Park J, et al. Human kidney tubule-specific gene expression based dissection of chronic kidney disease traits. EBioMedicine 2017;24:267-76.

12 Liu J, Kumar S, Dolzhenko E, et al. Molecular characterization of the transition from acute to chronic kidney injury following ischemia/ reperfusion. JCl Insight 2017;2:e94716.

13 Wu H, Lai C-F, Chang-Panesso M, et al. Proximal tubule translational profiling during kidney fibrosis reveals proinflammatory and long noncoding RNA expression patterns with sexual dimorphism. J Am Soc Nephrol 2020;31:23-38.

14 Kirita Y, Wu H, Uchimura K, et al. Cell profiling of mouse acute kidney injury reveals conserved cellular responses to injury. Proc Natl Acad Sci U S A 2020;117:15874-83.

15 Niewczas MA, Pavkov ME, Skupien J, et al. A signature of circulating inflammatory proteins and development of end-stage renal disease in diabetes. Nat Med 2019;25:805-13.

16 Greenberg JH, Abraham AG, Xu Y, et al. Plasma biomarkers of tubular injury and inflammation are associated with CKD progression in children. J Am Soc Nephrol 2020;31:1067-77.

17 Chen Y, Du X-Y. Functional properties and intracellular signaling of CCN1/Cyr61. J Cell Biochem 2007;100:1337-45.

$18 \mathrm{Kim} \mathrm{K-H}$, Won JH, Cheng N, et al. The matricellular protein CCN1 in tissue injury repair. J Cell Commun Signal 2018:12:273-9.

19 Bai T, Chen C-C, Lau LF. Matricellular protein CCN1 activates a proinflammatory genetic program in murine macrophages. $\mathrm{J}$ Immunol 2010;184:3223-32.

20 Lai C-F, Chen Y-M, Chiang W-C, et al. Cysteine-rich protein 61 plays a proinflammatory role in obstructive kidney fibrosis. PLoS One 2013;8:e56481.

21 Imhof BA, Jemelin S, Ballet R, et al. CCN1/CYR61-mediated meticulous patrolling by Ly6Clow monocytes fuels vascular inflammation. Proc Natl Acad Sci U S A 2016;113:E4847-56.

22 Muramatsu Y, Tsujie M, Kohda Y, et al. Early detection of cysteine rich protein 61 (CYR61, CCN1) in urine following renal ischemic reperfusion injury. Kidney Int 2002;62:1601-10.

23 Sawai K, Mukoyama M, Mori K, et al. Expression of CCN1 (CYR61) in developing, normal, and diseased human kidney. Am J Physiol Renal Physiol 2007;293:F1363-72.

24 Li C, Zhao L, Wang Y, et al. Cysteine-Rich protein 61, a specific ultraearly biomarker in kidney ischemia/reperfusion injury. Nephrology 2019;24:798-805.

25 Lai C-F, Lin S-L, Chiang W-C, et al. Blockade of cysteine-rich protein 61 attenuates renal inflammation and fibrosis after ischemic kidney injury. Am J Physiol Renal Physiol 2014;307:F581-92.

26 Levey AS, Coresh J, Greene T, et al. Expressing the modification of diet in renal disease study equation for estimating glomerular filtration rate with standardized serum creatinine values. Clin Chem 2007;53:766-72.

27 Hornung RW, Reed LD. Estimation of average concentration in the presence of Nondetectable values. Appl Occup Environ Hyg 1990;5:46-51.

28 Hewett P, Ganser GH. A comparison of several methods for analyzing censored data. Ann Occup Hyg 2007;51:611-32.

29 Li L, Chang A, Rostand SG, et al. A within-patient analysis for time-varying risk factors of CKD progression. J Am Soc Nephrol 2014;25:606-13.

30 Sheng X, Qiu C, Liu H, et al. Systematic integrated analysis of genetic and epigenetic variation in diabetic kidney disease. Proc Natl Acad Sci U S A 2020;117:29013-24.

31 Ramspek CL, de Jong Y, Dekker FW, et al. Towards the best kidney failure prediction tool: a systematic review and selection aid. Nephrol Dial Transplant 2020;35:1527-38.

32 McClellan WM, Flanders WD. Risk factors for progressive chronic kidney disease. J Am Soc Nephrol 2003;14:S65-70.

33 Kovesdy CP, Trivedi BK, Kalantar-Zadeh K, et al. Association of anemia with outcomes in men with moderate and severe chronic kidney disease. Kidney Int 2006;69:560-4.

34 Agarwal R, Duffin KL, Laska DA, et al. A prospective study of multiple protein biomarkers to predict progression in diabetic chronic kidney disease. Nephrol Dial Transplant 2014;29:2293-302.

35 Sabbisetti VS, Waikar SS, Antoine DJ, et al. Blood kidney injury molecule-1 is a biomarker of acute and chronic kidney injury and predicts progression to ESRD in type I diabetes. J Am Soc Nephrol 2014;25:2177-86.

36 Rysz J, Gluba-Brzózka A, Franczyk B, et al. Novel biomarkers in the diagnosis of chronic kidney disease and the prediction of its outcome. Int J Mol Sci 2017:18:1702.

37 Pena MJ, Stenvinkel P, Kretzler M, et al. Strategies to improve monitoring disease progression, assessing cardiovascular risk, and defining prognostic biomarkers in chronic kidney disease. Kidney Int Suppl 2017;7:107-13.

38 Rodríguez-Ortiz ME, Pontillo C, Rodríguez M, et al. Novel urinary biomarkers for improved prediction of progressive EGFR loss in early chronic kidney disease stages and in high risk individuals without chronic kidney disease. Sci Rep 2018;8:15940.

39 Wada T, Yokoyama H, Su SB, et al. Monitoring urinary levels of monocyte chemotactic and activating factor reflects disease activity of lupus nephritis. Kidney Int 1996;49:761-7.

40 Grams ME, Sang Y, Ballew SH, et al. Evaluating glomerular filtration rate slope as a surrogate end point for ESKD in clinical trials: an individual participant meta-analysis of observational data. J Am Soc Nephrol 2019;30:1746-55. 